\title{
Diálise Peritoneal nos Dois Primeiros Anos de Vida: Experiência de uma Unidade de Nefrologia e Transplantação Renal Pediátrica
}

\section{Peritoneal Dialysis in the First Two Years of Life: Experience of a Nephrology and Renal Transplantation Pediatric Unit}

\author{
Sofia DEUCHANDE¹, Tânia MANO², Cristina NOVAIS $\square^{3}$, Rute MACHADO ${ }^{4}$, Rosário STONE ${ }^{5}$, Margarida ALMEIDA $^{5}$
} Acta Med Port 2016 Sep;29(9):525-532 - http://dx.doi.org/10.20344/amp.6913

\section{RESUMO}

Introdução: A diálise peritoneal é o método dialítico de eleição perante doença renal crónica terminal em idade pediátrica. O objetivo deste estudo foi caracterizar a sobrevivência a longo prazo de uma população de crianças, que iniciou diálise peritoneal nos dois primeiros anos de vida.

Material e Métodos: Estudo descritivo e retrospetivo, realizado numa unidade de nefrologia e transplantação renal pediátrica portuguesa, no período de janeiro de 1991 a agosto de 2014. Avaliou-se etiologia da doença renal crónica terminal, mortalidade, comorbilidades e complicações da diálise peritoneal e da doença renal crónica terminal, crescimento e desenvolvimento psicomotor.

Resultados: Vinte crianças iniciaram diálise peritoneal antes dos dois anos. Ocorreram seis óbitos; não houve mortalidade em crianças com doença renal primária nos últimos 10 anos. Caracterizaram-se os 14 sobreviventes, 13 do sexo masculino. As anomalias congénitas do rim e do trato urinário constituíram a principal causa de doença renal crónica terminal (45\%).O início de diálise peritoneal ocorreu em média aos 6,1 meses, em seis casos antes dos 30 dias de vida. A peritonite foi o motivo mais frequente de internamento. Dez crianças foram transplantadas, com idade média de 5,3 anos. Em relação ao crescimento, as quatro crianças que se mantêm em diálise peritoneal têm baixa estatura, mas nove dos transplantados têm uma estatura final dentro do esperado para a sua estatura-alvo familiar. Nove (64\%) tiveram alterações no desenvolvimento psicomotor.

Discussão: A diálise peritoneal é uma técnica possível e exequível desde o nascimento, tal como evidenciado nesta amostra, em que se iniciou com sucesso em mais de metade das crianças antes dos seis meses de vida. Permite uma sobrevivência a longo prazo até à possibilidade do transplante renal apesar da morbilidade associada, nomeadamente as peritonites e as complicações da doença renal crónica. As dez crianças transplantadas desta amostra melhoraram o seu crescimento, recuperaram da anemia crónica e melhoraram da dislipidémia, comparativamente com o período em diálise. No entanto, o tempo médio de espera até ao TR de 5,3 anos foi superior ao de outros centros internacionais.

Conclusão: Estes dados apoiam a utilização da diálise peritoneal desde o nascimento, embora as complicações e o pior crescimento associados reflitam a necessidade de desenvolver estratégias para otimizar nutrição, crescimento e desenvolvimento e reduzir o tempo pré-transplante renal.

Palavras-chave: Diálise Peritoneal; Insuficiência Renal Crónica; Transplantação de Rim; Trato Urinário/anomalias congénitas.

\section{ABSTRACT}

Introduction: Peritoneal dialysis is the dialytic method of choice in chronic end-stage renal disease in children. This study main purpose was to characterize the long-term survival of a pediatric population who began peritoneal dialysis within the first two years of life.

Material and Methods: A descriptive and retrospective study was performed in a portuguese nephrology and renal transplantation pediatric unit, between January 1991 and August 2014. End-stage renal disease etiology, mortality, comorbidities and complications of peritoneal dialysis and end-stage renal disease, growth and psychomotor development were evaluated.

Results: Twenty children started peritoneal dialysis within the first two years of life. There were six deaths, but no deaths of children with primary chronic kidney disease were registered over the past decade. The 14 living children were characterized; 13 were males. Congenital abnormalities of the kidney and urinary tract were the leading etiology of chronic kidney disease (45\%). The average age start of peritoneal dialysis was 6.1 months; six children started before 30 days of life. Peritonitis was the most frequent cause of hospitalization. Ten children were transplanted at an average age of 5.3 years. All of the children who are still in peritoneal dialysis have short stature, but nine of the transplanted have final height within the expected for their mid-parental height target range. Nine (64\%) had some type of neurodevelopmental delay.

Discussion: Peritoneal dialysis is a technique possible and feasible since birth, as evidenced in the study, as more than half of children successfully started it before 6 months of life. It allows long-term survival until the possibility of renal transplantation despite the associated morbidity, including peritonitis and complications of chronic renal disease. The ten transplanted children improved their growth, recovered from chronic anemia and improved dyslipidemia, compared with the period of dialysis. However, the average waiting time until the renal transplant was 5.3 years higher than other international centers.

Conclusion: These data support the use of peritoneal dialysis from birth, but complications and the worst growth reflect the need to develop strategies to optimize care relating to nutrition, growth and development and to reduce pre-transplant time.

Keywords: Kidney Transplantation; Peritoneal Dialysis; Renal Insufficiency, Chronic; Urinary Tract/abnormalities.

1. Unidade de Pediatria. Departamento da Mulher e da Criança. Hospital José de Almeida. Cascais. Portugal.

2. Faculdade de Medicina. Universidade de Lisboa. Lisboa. Portugal.

3. Serviço de Pediatria. Hospital de Caldas da Rainha. Centro Hospitalar do Oeste. Caldas de Rainha. Portugal.

4. Departamento de Pediatria. Hospital de Santa Maria. Centro Hospitalar Lisboa Norte. Lisboa. Portugal.

5. Unidade de Nefrologia e Transplantação Renal Pediátrica. Departamento de Pediatria. Hospital de Santa Maria. Centro Hospitalar Lisboa Norte. Lisboa. Portugal.

$\triangle$ Autor correspondente: Cristina Novais. criscnovais@gmail.com

Recebido: 03 de março de 2015 - Aceite: 23 de fevereiro de 2016 | Copyright @ Ordem dos Médicos 2016 


\section{INTRODUÇÃO}

A diálise peritoneal (DP) é a modalidade de diálise preferencial em idade pediátrica, perante doença renal crónica terminal (DRCT), aplicável desde o nascimento. É a técnica com maior experiência de utilização na maioria das unidades de nefrologia pediátrica, permitindo uma melhor preservação da função renal residual e garantindo, pela possibilidade de utilização domiciliária, uma maior autonomia do doente e da sua família. A hemodiálise é menos usada em idade pediátrica, especialmente em idades muito jovens, pelas dificuldades associadas à própria técnica (acesso vascular permanente, elevado volume de sangue no circuito extracorporal, programas de diálise intensivos, ...) e pela necessidade de deslocamento a um centro de diálise. ${ }^{1,2}$

A DRCT é uma situação rara nos dois primeiros anos de vida. Os últimos dados epidemiológicos publicados do Registry of the European Society for Paediatric Nephrology, European Renal Association e European Dialysis and Transplantation Association (ESPN/ERA-EDTA), referentes a 2012, revelam uma incidência e prevalência em Portugal de crianças em terapêutica de substituição renal (TSR), do nascimento aos 14 anos, respetivamente de 10,2 e 53,8 por milhão de habitantes da mesma faixa etária (PMHFE), registando-se abaixo dos quatro anos uma prevalência de 20,6 PMHFE. 3,4

O grupo etário até aos dois anos constitui cerca de $12,5 \%$ a $47 \%$ da população pediátrica em diálise peritoneal (DP). ${ }^{4-6} \mathrm{~A}$ displasia renal é a etiologia de DRCT predominante nesta faixa etária. ${ }^{7-11} \mathrm{~A}$ DP neste grupo é um processo desafiante, continuando o seu início a motivar questões médicas e éticas. ${ }^{6,7,12,} \mathrm{~A}$ incidência de complicações da técnica é mais elevada, sendo as peritonites e as infeções associadas ao cateter as mais frequentes. $2,9,11,13,14$ A taxa de mortalidade abaixo dos dois anos é até 55 vezes mais elevada que noutras idades pediátricas, embora séries recentemente publicadas revelem uma mudança nesta tendência associada à melhoria nos cuidados. ${ }^{2,8,11,12} \mathrm{~A}$ mortalidade associa-se, mais do que à técnica, à presença de comorbilidades como cardiopatia, hipoplasia pulmonar e perturbações do desenvolvimento psicomotor. ${ }^{2,3,13} \mathrm{~A}$ prematuridade, secundária a doença nefrourológica, é outro fator que pode, pelas suas próprias exigências e especificidades, complicar o tratamento. ${ }^{10}$

A atuação na DRCT engloba também o controlo mineral ósseo e ácido-base, o tratamento da anemia e a otimização da nutrição e crescimento, com recurso, se necessário, a nutrição entérica ou parentérica, a uma dieta ajustada às necessidades, com suplementação de nutrientes, vitaminas e oligoelementos e hormona de crescimento. Uma adequada intervenção médica, cirúrgica, psicológica e social pode contribuir para melhorar o prognóstico a longo prazo destas crianças. Nesta população, destaca-se ainda a importância da prevenção de doença cardiovascular, cujo risco aumenta em função do tempo em diálise. . $2,5,15^{2}$

Nestes doentes, o principal objetivo é otimizar o estado clínico e nutricional e o crescimento, até que se reúnam as condições para um transplante renal (TR), que é o que permite oferecer as melhores condições de sobrevivência e qualidade de vida. ${ }^{1,2,9}$

Os objetivos primários deste trabalho foram caracterizar, a partir do estudo de uma população com DRCT que iniciou DP nos dois primeiros anos de vida, a sobrevivência a longo prazo até ao transplante renal, a etiologia da DRCT, as comorbilidades e as complicações da técnica de DP e da doença renal crónica. Os objetivos secundários foram analisar o crescimento estaturo-ponderal e as eventuais alterações do desenvolvimento psicomotor, com repercussão no aproveitamento escolar, da população em estudo.

\section{MATERIAL E MÉTODOS}

Foi realizado um estudo descritivo, retrospetivo, da população pediátrica com DRCT, seguida numa unidade de nefrologia pediátrica de um hospital universitário nível III, que iniciou DP com idade inferior a dois anos, no período de janeiro de 1991 até agosto de 2014.

A recolha de dados foi realizada mediante a consulta dos processos clínicos e por contacto telefónico com pais/ cuidadores.

Os dados colhidos incluíram:

- Caracterização da amostra: etiologia da DRCT; idade de início de DP e, se aplicável, idade de inicio de hemodiálise e TR; presença de anúria (diurese inferior a $0,5 \mathrm{~mL} / \mathrm{kg} / \mathrm{dia}$ ) e patologias associadas.

- Mortalidade (com determinação da causa dos óbitos)

- Morbilidade: número de internamentos em DP e principal motivo de internamento; complicações associadas à DP (peritonite); complicações associadas à DRCT: hipertensão arterial (critérios para hipertensão arterial segundo 4th Report on Diagnosis, Evaluation, and Treatment of High Blood Pressure in Children and Adolescents. 2004), anemia, ${ }^{16}$ hiperparatiroidismo grave (hormona paratiroideia $\geq 1000 \mathrm{mg} / \mathrm{dL}$ sem resposta à terapêutica convencional), dislipidémia e alteração do perfil glicídico.

- Avaliação do crescimento: somatometria (no início de DP, na data de TR e no final do seguimento), determinação da estatura-alvo familiar e do canal do crescimento (limites $10 \mathrm{~cm}$ acima e abaixo, correspondentes aos percentis três e 97 (limites superior e inferior) do canal de crescimento esperado para cada criança), ${ }^{17}$ tratamento com hormona de crescimento, ${ }^{16}$ índice de massa corporal (IMC), necessidade de nutrição entérica (sonda gastrointestinal, gastrostomia).

- Avaliação do desenvolvimento psicomotor, ano de escolaridade e necessidade de ensino especial.

A avaliação dos parâmetros laboratoriais baseou-se nos valores de referência do laboratório, de acordo com a idade e a situação clínica.

O cálculo do z-score da estatura e do IMC foi realizado mediante a aplicação do programa da Organização Mundial de Saúde (OMS) 2006/2007, com exceção dos recém-nascidos prematuros nos quais foi usado o modelo Fenton 
2013 para o período neonatal. ${ }^{17}$

A análise estatística descritiva foi realizada mediante a utilização do programa SPSS ${ }^{\circledR}$ v20.0.

Obteve-se autorização institucional da Comissão de Ética para a realização do estudo.

\section{RESULTADOS}

Durante o período do estudo um total de 20 crianças com idade inferior a dois anos iniciaram DP, 17 das quais do sexo masculino (85\%).

As anomalias congénitas dos rins e trato urinário (45\%, $\mathrm{n}=9$ ) e a lesão renal aguda (LRA) hipoxico-isquémica neonatal $(20 \%, n=4)$ constituíram as etiologias mais frequentes de DRCT nos doentes em estudo (Tabela 1).

Neste período ocorreram seis óbitos (30\%): quatro crianças com DRCT secundária a lesão renal aguda (LRA) hipoxico-isquémica faleceram por complicações de cardiopatia congénita, sépsis e insuficiência cardíaca; as restantes crianças, ambas nascidas antes de 1999, com DRCT primária, faleceram no contexto de sépsis e hipercaliemia grave. Não foi possível recolher dados adicionais sobre os doentes falecidos, pelo que foram excluídos da restante análise.

A restante análise incluiu 14 crianças, 13 do sexo masculino (93\%), num tempo médio de seguimento de $10 \pm 5,3$ anos (mediana:10; 1,9 - 20 anos). No final do estudo, a média de idades foi de 10,5 \pm 5 anos (mediana 10; 3 - 20 anos).

O início da DP ocorreu, em média, aos 6,1 meses de vida tendo oito das crianças (57\%) iniciado DP antes dos seis meses, seis $(43 \%)$ delas antes do primeiro mês de vida.

Todos os doentes realizaram diálise automatizada na modalidade noturna intermitente, por vezes com ciclo diurno.

Em relação às patologias associadas destacou-se a presença de prematuridade em seis crianças (43\%), cardiopatia congénita minor em quatro crianças (29\%) (comunicação interauricular, comunicação interventricular e insuficiência mitral minor), hipotiroidismo em cinco (36\%) e uma criança com síndrome de Pierson, que além da síndrome nefrótica congénita e alterações oculares sindromáticas, tinha também epilepsia e laringomalácia.

Relativamente à diurese, do total de 14 crianças, nove tiveram critérios de anúria durante o período englobado pelo estudo. Das quatro crianças que se mantêm em DP, três encontram-se em anúria.

As restantes crianças $(n=10 ; 71 \%)$ foram submetidas a transplante renal (TR). Apenas uma das crianças necessitou transitoriamente de hemodiálise antes do TR. A média de tempo de espera até ao TR foi de cinco anos $(1,7-7,2$ anos) e a idade média do TR foi de 5,3 \pm 1,6 anos $(2,1-7,7$ anos). A sobrevida atual dos enxertos é de $100 \%$, com uma média de tempo pós-transplantação de 7,3 anos (1,4 - 18,4 anos), estando duas crianças no estádio I, sete no II e uma no III da DRC-T da classificação da Kidney Disease Outcomes Quality Initiative. Nenhuma das crianças recebeu transplante de dador vivo.

Tabela 1 - Etiologia da doença renal crónica terminal e mortalidade dos 20 doentes que iniciaram diálise peritoneal antes dos dois anos de vida

\begin{tabular}{|c|c|c|c|}
\hline $\begin{array}{l}\text { TOTAL } \\
\mathrm{n}(\%)\end{array}$ & $\begin{array}{c}\text { Vivos } \\
\mathrm{n}=14(70 \%)\end{array}$ & $\begin{array}{c}\text { Óbitos } \\
n=6(30 \%)\end{array}$ & $\begin{array}{c}\text { TOTAL } \\
\mathrm{n}=20(100 \%)\end{array}$ \\
\hline Anomalias congénitas dos rins e do trato urinário & $7(50 \%)$ & $2(33 \%)$ & $9(45 \%)$ \\
\hline \multicolumn{4}{|l|}{ Doenças genéticas } \\
\hline Síndrome nefrótica congénita (síndrome de Pierson) & $2(14,15 \%)$ & 0 & $2(10 \%)$ \\
\hline Disgenesia tubular renal e do sistema renina-angiotensina & $1(7,1 \%)$ & 0 & $1(5 \%)$ \\
\hline Doença renal glomeruloquística bilateral & $1(7,1 \%)$ & 0 & $1(5 \%)$ \\
\hline Glomerulonefrite crescêntica de etiologia desconhecida & $1(7,1 \%)$ & 0 & $1(5 \%)$ \\
\hline Síndroma hemolítico-urémico & $2(14,1 \%)$ & 0 & $2(10 \%)$ \\
\hline Lesão renal aguda secundária a hipóxia-isquemia & 0 & $4(67 \%)$ & $4(20 \%)$ \\
\hline
\end{tabular}

Tabela 2 - Avaliação do z-score da estatura das quatro crianças que se mantinham em diálise peritoneal no final do estudo: ao nascimento, no início da diálise peritoneal e no final do estudo (segundo as curvas de crescimento da OMS e curvas de Fenton nos recém-nascidos pré-termo)

\begin{tabular}{cccccc}
\hline $\begin{array}{c}\text { Idade gestacional } \\
\text { (semanas) }\end{array}$ & $\begin{array}{c}\text { Z-score Estatura } \\
\text { - Nascimento }\end{array}$ & $\begin{array}{c}\text { Idade (meses) } \\
\text { - Início DP }\end{array}$ & $\begin{array}{c}\text { Z-score Estatura } \\
- \text { Início DP }\end{array}$ & $\begin{array}{c}\text { Idade (anos) } \\
\text { - Final estudo }\end{array}$ & $\begin{array}{c}\text { Z-score Estatura } \\
\text { - Final estudo }\end{array}$ \\
\hline 35 & $-1,6^{*}$ & 15,01 & -5 & 3,4 & $-3,6$ \\
35 & $0,6^{*}$ & 21,09 & $-5,3$ & 5,75 & $-2,83$ \\
38 & 0,06 & 5,36 & $-2,6$ & 9,4 & $-4,13$ \\
40 & $-0,5$ & 8,97 & 0,5 & 3,08 & $-2,3$ \\
\hline
\end{tabular}

DP: Diálise peritoneal; * Z-score avaliado segundo as curvas de Fenton 
Tabela 3 - Avaliação do z-score da estatura das dez crianças com TR: ao nascimento, no início da diálise peritoneal, à data do transplante renal e no final do estudo. (segundo as curvas de crescimento da OMS e curvas de Fenton nos recém-nascidos pré-termo)

\begin{tabular}{|c|c|c|c|c|c|c|c|}
\hline $\begin{array}{c}\text { Idade } \\
\text { Gestacional } \\
\text { (semanas) }\end{array}$ & $\begin{array}{l}\text { Z-score Estatura } \\
\text { - Nascimento }\end{array}$ & $\begin{array}{l}\text { Idade } \\
\text { - Início DP }\end{array}$ & $\begin{array}{l}\text { Z-score } \\
\text { Estatura } \\
\text { - Início DP }\end{array}$ & $\begin{array}{c}\text { Idade } \\
\text { TR } \\
\text { (anos) }\end{array}$ & $\begin{array}{c}\text { Z-score } \\
\text { Estatura } \\
\text { - TR }\end{array}$ & $\begin{array}{c}\text { Idade (anos) } \\
\text { - Final } \\
\text { estudo }\end{array}$ & $\begin{array}{c}\text { Z-score } \\
\text { Estatura } \\
\text { - Final estudo }\end{array}$ \\
\hline 32 & 0,1 * & 15 dias & 0,1 * & 5,59 & -2 & 9,91 & $-0,9$ \\
\hline 36 & $-1,7^{*}$ & 2 dias & $-1,7^{*}$ & 4,83 & $-1,7$ & 15,41 & $-1,9$ \\
\hline 36 & $-0,2$ * & 4 dias & $-0,2$ * & 4,83 & $-4,3$ & 10,16 & $-1,4$ \\
\hline 36 & 0,3 * & 11,1 meses & 1,43 & 4,33 & $-1,9$ & 5,79 & $-2,4$ \\
\hline 37 & 0,47 & 8 dias & $-1,2$ & 4 & $-1,5$ & 7 & $-1,5$ \\
\hline 37 & $-3,64$ & 10 dias & $-4,1$ & 7,23 & $-1,3$ & 14,25 & $-3,1$ \\
\hline 38 & Desconhecido & 10,2 meses & Desconhecido & 6,72 & $-3,3$ & 17 & $-4,1$ \\
\hline 38 & $-2,05$ & 5 dias & $-2,5$ & 5,37 & $-1,4$ & 11,38 & $-1,1$ \\
\hline 39 & $-0,47$ & 7,23 meses & Desconhecido & 7,66 & $-4,2$ & 14,24 & $-4,4$ \\
\hline 41 & 1,7 & 4,57 meses & 1,4 & 2,1 & $-2,2$ & 20,25 & $-1,58$ \\
\hline
\end{tabular}

DP: Diálise peritoneal; TR: Transplante renal; ${ }^{*}$ Z-score avaliado segundo as curvas de Fenton

Em relação ao número de internamentos, durante o período do estudo, verificou-se uma média de 2,6 internamentos/ano/doente, com maior número de internamentos durante o período de diálise DP em relação ao período pós-transplante (2,9 versus 1,8 internamentos/ano/doente). 0 motivo mais frequente de internamento foi a peritonite. Os restantes motivos de internamento incluíram: início de técnica de DP, substituição de cateter de DP, intercorrências infeciosas ou otimização de diálise por estados de hipervolémia.

As peritonites foram a complicação mais frequente relacionada com a DP e ocorreram em $78 \%$ dos casos (11 crianças), com uma média de 0,91 episódios/doente/ano; três das crianças com duas ou mais peritonites/doente/ano.

Relativamente às complicações associadas à DRCT referem-se por ordem de frequência:

- A anemia crónica, foi a alteração mais frequente, universalmente presente em todas as 14 crianças durante o período em DP. Todas fizeram terapêutica com fatores de crescimento de eritropoiese e 11 crianças (78\%) necessitaram pelo menos uma vez de suporte transfusional. A criança em DP há mais tempo no final do estudo ( 9,4 anos) teve períodos de anemia refratária ao tratamento com necessidade frequente de transfusões. No período pós-transplante, houve recuperação da anemia em sete $(70 \%)$ crianças.

- As complicações cardiovasculares, destacando a prevalência de hipertensão arterial (HTA) em 12 crianças (86\%), com uma duração média de $6,9 \pm 5,1$ anos $(1,3$ e 19,4 anos); em três (21\%) delas verificou-se resolução da HTA pós-transplante. Identificou-se hipertrofia ventricular esquerda (HVE) durante algum período do estudo em cinco crianças hipertensas (36\%), com regressão completa após tratamento. Verificaram-se também, durante este período, dois casos de trombo- se venosa profunda (14\%).

- A dislipidémia que foi detetada em oito crianças (57\%), com uma duração média 5,4 \pm 3,1 anos (1,7 - 10,2 anos); três das crianças melhoraram o perfil lipídico pós-transplante.

- O hiperparatiroidimo grave durante o período em DP foi detetado em cinco doentes (36\%). Quatro destas crianças estiveram pelo menos quatro anos em DP.

- Nenhuma das crianças teve alterações do perfil glicídico (HbA1c normal), antes ou após TR.

Relativamente ao estudo do crescimento, verificou-se que as quatro crianças em DP no final do estudo mantiveram critérios de baixa estatura (inferior ao percentil 3 ou inferior a -2 desvio-padrão (SDS)) para a população normal nas curvas de crescimento da OMS, embora em três delas tenha ocorrido uma ligeira recuperação da estatura ao longo do tempo, para valores de SDS ainda inferiores a -2SDS mas mais próximos deste (Tabela 2). A criança com maior tempo de diálise (9,4 anos) e síndrome de Pierson corresponde à situação mais grave (-4SDS).

Das dez crianças transplantadas, seis tinham, no final do estudo, uma estatura normal, mas quatro não recuperaram para valores superiores a -2SDS (Tabela 3 ).

A determinação do canal de crescimento revelou que todas as crianças em DP mantiveram estatura final inferior ao esperado, porém nove das 10 crianças transplantadas tinham estatura final dentro dos limites da respetiva estatura-alvo familiar (Tabela 4). O tratamento com hormona de crescimento foi efetuado em doze crianças (86\%).

$\mathrm{Na}$ avaliação do IMC detetaram-se apenas duas crianças em DP no final do estudo com um IMC inferior a -2 SDS para a população normal segundo a curva de crescimento da OMS, sendo que, a situação mais grave correspondia à criança há mais tempo em DP (9,4 anos) (Tabela 5). A maioria das crianças transplantadas $(90 \%$, ou seja, nove 
Tabela 4 - Comparação, em cada um dos 14 sobreviventes, entre o percentil da estatura final e os limites inferior e superior do canal de crescimento respetivo

\begin{tabular}{|c|c|c|c|}
\hline \multirow{2}{*}{$\begin{array}{c}\text { TOTAL } \\
(n=14)\end{array}$} & \multicolumn{2}{|c|}{ Canal de crescimento } & \multirow{2}{*}{$\begin{array}{l}\text { Percentil da } \\
\text { Estatura } \\
\text { - Final estudo }\end{array}$} \\
\hline & $\begin{array}{l}\text { Limite } \\
\text { inferior }\end{array}$ & $\begin{array}{l}\text { Limite } \\
\text { superior }\end{array}$ & \\
\hline DP & P3 & P85 & $<\mathrm{P} 3$ \\
\hline DP & P3 & P85 & $<\mathrm{P} 3$ \\
\hline DP & P3 & P97 & $<\mathrm{P} 3$ \\
\hline DP & P15 & > P97 & $<\mathrm{P} 3$ \\
\hline TR & P3 & P85 & $<\mathrm{P} 3$ \\
\hline TR & P3 & P85 & P15 \\
\hline TR & $<\mathrm{P} 3$ & P50 & P3 \\
\hline TR & P3 & P97 & P3 - 15 \\
\hline TR & $<\mathrm{P} 3$ & P50 & $<\mathrm{P} 3$ \\
\hline TR & P3 & P85 & P3 - 15 \\
\hline TR & $<\mathrm{P} 3$ & P50 & $<\mathrm{P} 3$ \\
\hline TR & P3 & P97 & P3 - 15 \\
\hline TR & $<\mathrm{P} 3$ & P85 & $\mathrm{P} 15$ \\
\hline TR & $<\mathrm{P} 3$ & P50 & $<\mathrm{P} 3$ \\
\hline
\end{tabular}

P: Percentil; DP: Diálise peritoneal; TR: Transplante renal

das 14) teve um IMC dentro dos valores normais no final do estudo (Tabela 6). Nove crianças necessitaram de nutrição entérica, oito por sonda nasogástrica (57\%) e uma criança por enterostomia percutânea $(7 \%)$.

Em relação ao desenvolvimento psicomotor (DPM), nove crianças (64\%) tiveram diagnóstico de atraso do DPM, em algum momento do estudo. Não foi possível, no entanto, especificar o perfil funcional completo destas crianças a partir da consulta dos processos clínicos. Cinco crianças necessitaram de ensino especial. No final do estudo, 10 crianças encontravam-se em idade escolar, sendo que oito frequentavam o ensino regular e um adolescente o ensino superior; três crianças nunca reprovaram.

\section{DISCUSSÃO}

Neste grupo de crianças que iniciaram DP com idade inferior a dois anos as etiologias mais frequentes de DRCT são as mais referidas na literatura ${ }^{2-9}$ : anomalias congénitas dos rins e trato urinário e DRCT secundária a lesão renal aguda grave resultante de situações de hipoxia-isquemia neonatais. As causas genéticas identificadas, raras e pontuais, foram de expressão clínica precoce, tal como tem sido relatado noutros estudos.

A preponderância do género masculino na nossa amostra está de acordo com o que já foi demonstrado noutras publicações. ${ }^{18,19} \mathrm{O}$ número de prematuros pode estar associado à elevada prevalência de patologia congénita nefrourológica, que constitui um fator de risco para prematuridade. ${ }^{10}$

Relativamente aos óbitos, encontraram-se causas que mais frequentemente se associam a mortalidade em crianças com DRCT no primeiro ano de vida, a doença cardíaca ou infeciosa. ${ }^{12,13}$ Nesta amostra a mortalidade global foi de $30 \%$, embora, tal como descrito na literatura, ${ }^{6,8,9,15,19-21}$ se tenha notado uma melhoria desta tendência na última década do estudo e não se tenham registado óbitos de crianças com DRCT primária. A mortalidade em crianças que iniciam DP antes dos dois anos é até quatro vezes superior à encontrada em crianças mais velhas. ${ }^{6,8,9,15,19-21} \mathrm{Em}$ séries europeias e norte-americanas publicadas, a taxa de sobrevivência, após um ano em DP, em crianças com idade inferior a dois anos é de cerca de $85 \%$, comparativamente com uma taxa de $95 \%$ em crianças que iniciaram DP com idade superior a dois anos., ${ }^{3,5,6,8,22}$ No entanto, alguns estudos descrevem que em crianças em DP com idade inferior a dois anos sem comorbilidades associadas, a sobrevida é semelhante à das crianças mais velhas. ${ }^{10,13,14,23}$

Relativamente à DP, oito das 14 crianças (57\%) iniciaram diálise antes dos seis meses de vida, seis delas $(43 \%$ do total das 14) no primeiro mês de vida. Todas as crianças realizaram dialise automatizada noturna intermitente, o que permite uma maior autonomia do doente e da sua família, conduzindo a uma maior aceitação. Apesar de esta modalidade contribuir para preservar a função renal residual, metade das crianças apresentaram deterioração da função renal residual com instalação progressiva de anúria, possivelmente em relação com tempo prolongado em diálise e peritonites. A presença de anúria agrava o prognóstico, aumentando 7,5 vezes o risco de morte..$^{9,14,23}$

Relativamente às dez crianças transplantadas, verificou-se um impacto positivo na nossa amostra com

Tabela 5 - Avaliação do z-score do índice de massa corporal das quatro crianças que se mantinham em diálise peritoneal no final do estudo: ao nascimento, no início da diálise peritoneal, à data do transplante renal e no final do estudo (segundo as curvas de crescimento da OMS).

\begin{tabular}{cccccc}
\hline $\begin{array}{c}\text { Idade Gestacional } \\
\text { (semanas) }\end{array}$ & $\begin{array}{c}\text { Z-score IMC } \\
\text { - Nascimento }\end{array}$ & $\begin{array}{c}\text { Idade (meses) } \\
\text { - Início DP }\end{array}$ & $\begin{array}{c}\text { Z-score IMC } \\
\text { - Início DP }\end{array}$ & $\begin{array}{c}\text { Idade (anos) } \\
\text { - Final estudo }\end{array}$ & $\begin{array}{c}\text { Z-score IMC } \\
- \text { Final estudo }\end{array}$ \\
\hline 35 & $-1,16$ & 15,01 & $-1,96$ & 3,4 & $-0,61$ \\
35 & $-1,33$ & 21,09 & $-0,15$ & 5,75 & $-2,83$ \\
38 & $-1,51$ & 5,36 & $-3,86$ & 9,4 & $-4,26$ \\
40 & 1,04 & 8,97 & $-1,22$ & 3,08 & 1,8 \\
\hline
\end{tabular}

IMC: Índice de massa corporal; DP: Diálise peritoneal 
Tabela 6 - Avaliação do z-score do índice de massa corporal das dez crianças com transplante renal: ao nascimento, no início da diálise peritoneal, à data do transplante renal e no final do estudo. (segundo as curvas de crescimento da OMS)

\begin{tabular}{|c|c|c|c|c|c|c|c|}
\hline $\begin{array}{c}\text { Idade } \\
\text { Gestacional } \\
\text { (semanas) }\end{array}$ & $\begin{array}{l}\text { Z-score IMC } \\
\text { - Nascimento }\end{array}$ & $\begin{array}{l}\text { Idade } \\
\text { - Início DP }\end{array}$ & $\begin{array}{l}\text { Z-score IMC } \\
\text { - Início DP }\end{array}$ & $\begin{array}{l}\text { Idade TR } \\
\text { (anos) }\end{array}$ & $\begin{array}{c}\text { Z-score IMC } \\
\text { - TR }\end{array}$ & $\begin{array}{l}\text { Idade (anos) } \\
\text { - Final estudo }\end{array}$ & $\begin{array}{l}\text { Z-score IMC } \\
\text { - Final estudo }\end{array}$ \\
\hline 32 & $-5,09$ & 15 dias & Desconhecido & 5,59 & 0,97 & 9,91 & 0,76 \\
\hline 36 & $-2,6$ & 2 dias & $-2,6$ & 4,83 & 1,7 & 15,41 & 0,42 \\
\hline 36 & $-1,76$ & 4 dias & $-1,59$ & 4,83 & 1,59 & 10,16 & 0,61 \\
\hline 36 & 1,14 & 11,1 meses & Desconhecido & 4,33 & 0,03 & 5,79 & $-0,38$ \\
\hline 37 & 0,23 & 8 dias & $-0,04$ & 4 & $-0,44$ & 7 & $-0,13$ \\
\hline 37 & $-0,25$ & 10 dias & $-1,39$ & 7,23 & $-0,5$ & 14,25 & $-0,76$ \\
\hline 38 & Desconhecido & 10,2 meses & Desconhecido & 6,72 & $-0,63$ & 17 & 1,15 \\
\hline 38 & $-0,97$ & 5 dias & $-0,89$ & 5,37 & 0,56 & 11,38 & 0,96 \\
\hline 39 & $-0,64$ & 7,23 meses & Desconhecido & 7,66 & $-2,82$ & 14,24 & $-0,14$ \\
\hline 41 & 2,65 & 4,57 meses & Desconhecido & 2,1 & $-0,45$ & 20,25 & $-2,45$ \\
\hline
\end{tabular}

IMC: Índice de massa corporal; DP: Diálise peritoneal; TR: Transplante renal

melhoria no crescimento, recuperação da anemia e dislipidémia e redução da morbilidade, comparativamente com o período em diálise. No entanto, o tempo médio de espera até ao TR de 5,3 anos foi superior ao de outros centros internacionais. Fatores como os critérios da equipa cirúrgica envolvida no TR no nosso hospital (por exemplo, peso mínimo para TR de $12 \mathrm{~kg}$ ) ou a ausência de disponibilidade de dador vivo podem ter sido impeditivos de uma TR mais precoce. A transplantação renal em crianças com menos de cinco anos, particularmente se preemptive, tem demonstrado reduzir a mortalidade e melhorar o seu crescimento. .0,24 $^{10}$

Em relação às complicações relacionadas com a DP, a peritonite foi a complicação mais frequente e o principal motivo de internamento, tal como referido noutras séries. Apesar dos avanços tecnológicos e da melhoria dos cuidados de assepsia do cateter de DP, as peritonites continuam a ser a complicação mais comum em crianças em DP, sendo uma causa importante de morbilidade, hospitalização e falência da técnica. ${ }^{2,25,26}$ Tendo em conta que infeções recorrentes e graves podem resultar num comprometimento da função da membrana dialítica e fibrose peritoneal, deve ser instituído tratamento precoce e implementar medidas que promovam a sua prevenção, reforçando o ensino aos pais/cuidadores sobre cuidados de assepsia com o catéter. ${ }^{25,26}$

Em relação às complicações relacionadas com a DRCT, a mais frequente foi a anemia crónica, presente em todas as crianças da amostra. A anemia agrava o prognóstico e contribui para a diminuição da qualidade de vida. A necessidade de transfusões, além de contribuir para a progressão da HVE, induz sensibilização antigénica o que prejudica a possibilidade de um futuro transplante. A terapêutica com estimuladores da eritropoiese, uma das conquistas das últimas décadas, tem impacto positivo no prognóstico, pela redução drástica da necessidade transfusional. Após o TR a supressão medular associada à imunossupressão e as infeções virais podem perpetuar a anemia, como se verifi- cou em três das crianças transplantadas. . $27,28^{27}$

Relativamente às complicações cardiovasculares, a HTA muito prevalente neste grupo (85\%) e a HVE, diagnosticada em $36 \%$ das crianças, agravam o prognóstico cardiovascular e obrigam a uma abordagem terapêutica rigorosa. A doença cardiovascular, no contexto de DRCT em diálise, associa-se a lesões vasculares aceleradas e a cardiomiopatia prematura, sendo a principal causa de mortalidade das crianças com DRC, 2,9,29,30 apesar de nestas existir menor exposição aos fatores de risco cardiovascular da população adulta (diabetes mellitus, tabagismo, obesidade). Os fatores envolvidos na sua patogénese em idade pediátrica (doença mineral óssea, HTA, sobrecarga hídrica, anemia, dislipidémia, hipoalbuminémia, hiperhomocisteinemia, inflamação e stress oxidativo $)^{8}$ conduzem a disfunção endotelial, rigidez arterial e calcificação vascular e dos tecidos, contribuindo para o remodelling cardíaco e HVE. ${ }^{2,9,29,30} \mathrm{O}$ TR, ao restabelecer a função renal, contribui para a regressão parcial da arteriopatia e da HVE, o que se verificou na maioria das crianças submetidas a transplante nesta amostra. ${ }^{31}$

Neste estudo, quatro das cinco crianças em que foram detetados períodos de hiperparatiroidismo grave mantiveram-se em DP mais de quatro anos. Sabe-se que o risco desta complicação aumenta por cada ano de diálise, revertendo após o TR. ${ }^{32}$ É necessário um adequado controlo para evitar níveis muito elevados de PTH que conduzem a doença mineral-óssea (com estímulo da atividade osteoblástica e da remodelação óssea com consequências no crescimento), e níveis demasiadamente suprimidos que levem a doença adinâmica do osso. Ambas as situações também aumentam o risco cardiovascular levando a calcificações metastáticas vasculares e dos tecidos moles. ${ }^{12}$

A dislipidémia foi diagnosticada em mais de metade das crianças do nosso estudo. O início de DP em idade precoce associa-se frequentemente a alterações do perfil lipídico, o que é fator de risco para a aterosclerose. ${ }^{31}$ 
Nenhuma das crianças apresentou alterações do metabolismo glicídico, mesmo após TR, apesar da crescente incidência descrita de diabetes de novo pós-transplante (NODAT). Da mesma maneira, a obesidade não foi detetada nas crianças transplantadas, apesar do aumento de incidência descrito nas últimas décadas na população pediátrica. ${ }^{24,33,34}$ Estas diferenças podem dever-se à dispersão do estudo ao longo de 25 anos, período temporal em que os esquemas de imunossupressão e hábitos alimentares sofreram modificações.

Em relação ao crescimento, no final do estudo, verificou-se baixa estatura em oito crianças: as quatro crianças em DP e quatro das 10 crianças transplantadas. Todas as crianças que se mantinham ainda em DP mantiveram baixa estatura, embora três com recuperação parcial, o que reflete a dificuldade na otimização dos cuidados destes doentes. Sabe-se que a baixa estatura é umas das sequelas major a longo prazo da DRCT nesta faixa etária, com repercussões no bem-estar psicológico, qualidade de vida e integração social na idade adulta. ${ }^{2}$ No grupo estudado, a maioria das crianças em DP realizaram terapêutica com hormona de crescimento a partir dos dois anos de idade, o que está de acordo com vários estudos publicados que apoiam a utilização desta terapêutica quando a otimização da nutrição e o controlo clínico e metabólico são insuficientes. ${ }^{35-37}$ Por outro lado, a dificuldade na recuperação ponderal e do IMC notada na maioria dos doentes foi mais grave naqueles que se encontravam há mais tempo em DP, refletindo a dificuldade na sua otimização nutricional. ${ }^{12}$

Após o TR, a recuperação do crescimento foi pior do que o esperado, com quatro das 10 crianças transplantadas mantendo baixa estatura, resultados que poderão relacionar-se com a presença de comorbilidades, a deficiente otimização nutricional, a corticoterapia e o maior tempo de espera até ao TR comparativamente com outras séries publicadas. ${ }^{15}$ Apesar disto, analisando a estatura-alvo familiar e o canal de crescimento estimado para cada criança, verificou-se que todas as crianças transplantadas, com exceção de uma, se encontravam dentro dos limites de crescimento esperado para a altura dos seus pais.

Por fim, relativamente ao neurodesenvolvimento, na amostra estudada, a maioria das crianças frequentava o ensino regular, sendo que a presença, em algumas delas, de atraso de desenvolvimento psicomotor e de perturbações da aprendizagem estarão também provavelmente em relação com outros fatores não avaliados no âmbito deste estudo. Nas últimas décadas, está descrita uma melhoria do prognóstico do neurodesenvolvimento das crianças com DRCT em DP, relacionada com a melhoria dos cuidados. ${ }^{6,23}$ Estudos experimentais sugerem um efeito direto tóxico da uremia no crescimento e maturação cerebral e, uma vez que metade do crescimento cerebral pós-natal ocorre no primeiro ano de vida, o estabelecimento de DRCT em idade precoce pode resultar em lesão cerebral irreversível. ${ }^{38}$ No entanto, vários estudos demonstraram que crianças que iniciaram DP nos dois primeiros anos de vida e foram submetidos posteriormente a TR conseguiram alcançar um nível cognitivo normal e a frequência do ensino regular, sem necessidade de apoios especiais. ${ }^{4}$

O estudo realizado apresenta algumas limitações, nomeadamente pelo facto de se tratar de um estudo retrospetivo realizado com base numa amostra pequena de um único centro, o que não permitiu efetuar estudos estatísticos comparativos, nem identificar fatores preditores de evolução desfavorável, a longo prazo. Além disso, ao abranger um longo período de tempo, durante o qual ocorreu um avanço científico e tecnológico significativo nesta área, poderá ter havido uma heterogeneidade na abordagem destes doentes que não foi valorizada.

\section{CONCLUSÃO}

A DP é uma técnica exequível nos dois primeiros anos de vida, tal como verificado neste grupo de doentes, podendo ser iniciada desde o nascimento e permitindo a sobrevivência a longo prazo até à possibilidade de TR. As complicações e o pior crescimento associados à diálise motivam a procura de melhores estratégias de otimização dos cuidados, da nutrição, do controlo dos fatores de risco cardiovasculares e do reforço do apoio psicossocial. A transplantação renal em idade mais precoce, reduzindo-se o tempo de espera pré-transplante, tal como se observa noutros centros internacionais, teria um impacto positivo adicional no prognóstico a longo prazo.

\section{PROTECÇÃO DE PESSOAS E ANIMAIS}

Os autores declaram ter obtido autorização institucional da Comissão de Ética para a realização do estudo e que os procedimentos seguidos estavam de acordo com a Declaração de Helsínquia da Associação Médica Mundial.

\section{CONFIDENCIALIDADE DOS DADOS}

Os autores declaram ter seguido os protocolos do seu centro de trabalho acerca da publicação dos dados de doentes.

\section{CONFLITO DE INTERESSES}

Os autores declaram não ter nenhum conflito de interesses relativamente ao presente artigo.

\section{FONTES DE FINANCIAMENTO}

Não existiram fontes externas de financiamento para a realização deste artigo.

\section{REFERÊNCIAS}

1. Zurowska AM, Fischbach M, Watson AR, Edefonti A, Stefanidis CJ. Clinical practice recommendations for the care of infants with stage 5 chronic kidney disease (CKD5). Pediatr Nephrol. 2013;28:1739-48.

2. Warady BA, Neu AM, Schaefer F. Optimal care of the infant, child, and

adolescent on dialysis: 2014 update. Am J Kidney Dis. 2014;64:128-42. 3. Schaefer F, Groothoff JW. ESPN/ERA-EDTA Registry. Pediatric Data 2012. 2014.

4. Chesnaye N, Bonthuis M, Schaefer F, Groothoff JW, Verrina E, Heaf 
JG, et al. Demographics of paediatric renal replacement therapy in Europe : a report of the ESPN / ERA - EDTA registry. Pediatr Nephrol. 2014;29:2403-10.

5. North American Pediatric Renal Trials and Collaborative Studies. NAPRTCS 2011 Annual Dialysis Report. Vancouver: Emmes; 2011.

6. Rönnholm KA, Holmberg C. Peritoneal dialysis in infants. Pediatr Nephrol. 2006;21:751-6.

7. Harambat J, van Stralen KJ, Kim JJ, Tizard EJ. Epidemiology of chronic kidney disease in children. Pediatr Nephrol. 2012:363-73.

8. Jander A, Makulska I, Latoszyńska J, Boguszewska-Bączkowska $\mathrm{H}$, Bałasz-Chmielewska I, Zagożdżon I, et al. Successes and pitfalls of chronic peritoneal dialysis in infants - a Polish nationwide outcome study. Arch Med Sci. 2010;6:414-9.

9. Shroff R, Ledermann S. Long-term outcome of chronic dialysis in children. Pediatr Nephrol. 2009;24:463-74.

10. Wedekin M, Ehrich JH, Offner G, Pape L. Renal replacement therapy in infants with chronic renal failure in the first year of life. Clin J Am Soc Nephrol. 2010;5:18-23.

11. Carey WA, Talley LI, Sehring SA, Jaskula JM, Mathias RS. Outcomes of dialysis initiated during the neonatal period for treatment of end-stage renal disease: a North American Pediatric Renal Trials and Collaborative Studies special analysis. Pediatrics. 2007;119:e468-73.

12. Van Stralen KJ, Borzych-Dużalka D, Hataya H, Kennedy SE, Jager KJ, Verrina E, et al. Survival and clinical outcomes of children starting renal replacement therapy in the neonatal period. Kidney Int. 2014;86:168-74.

13. Kari JA, Gonzalez C, Ledermann SE, Shaw V, Rees L. Outcome and growth of infants with severe chronic renal failure. Kidney Int. 2000;57:1681-7.

14. Mekahli D, Shaw V, Ledermann SE, Rees L. Long-term outcome of infants with severe chronic kidney disease. Clin J Am Soc Nephrol. 2010;5:10-7.

15. Vidal E, Edefonti A, Murer L, Gianoglio B, Maringhini S, Pecoraro C, et al. Peritoneal dialysis in infants: the experience of the Italian Registry of Paediatric Chronic Dialysis. Nephrol Dial Transplant. 2012;27:388-95.

16. Tanner JM, Goldstein H, Whitehouse RH. Standards for children's height at ages 2-9 years allowing for height of parents. Arch Dis Child. 1970;45:755-62.

17. Fenton TR, Kim JH. A systematic review and meta-analysis to revise the Fenton growth chart for preterm infants. BMC Pediatr. 2013;13:59.

18. Rheault MN, Rajpal J, Chavers B, Nevins TE. Outcomes of infants $<28$ days old treated with peritoneal dialysis for end-stage renal disease. Pediatr Nephrol. 2009;24:2035-9.

19. Alexander RT, Foster BJ, Tonelli MA, Soo A, Nettel-Aguirre A Hemmelgarn BR, et al. Survival and transplantation outcomes of children less than 2 years of age with end-stage renal disease. Pediatr Nephrol. 2012;27:1975-83.

20. Tsai HL, Yang LY, Chin TW, Wang HH, Liu CS, Wei CF, et al. Outcome and risk factors for mortality in pediatric peritoneal dialysis. Perit Dial Int. 2010;30:233-9.

21. Neu AM, Sander A, Borzych-Duzalka D, Watson AR, Vallés PG, Ha IS, et al. Comorbidities in chronic pediatric peritoneal dialysis patients: a report of the International Pediatric Peritoneal Dialysis Network. Perit Dial Int. 2012;32:410-8.

22. Rees L. Management of the neonate with chronic renal failure. Semin
Fetal Neonatal Med. 2008:13:181-8.

23. Rees L. Long-term peritoneal dialysis in infants. Perit Dial Int. 2007;27:25-9.

24. Kim J, Marks S. Long-term outcomes of children after solid organ transplantation. Clinics. 2014;69:S28-38.

25. Warady BA, Feneberg R, Verrina E, Flynn JT, Müller-Wiefel DE, Besbas $\mathrm{N}$, et al. Peritonitis in children who receive long-term peritoneal dialysis: a prospective evaluation of therapeutic guidelines. J Am Soc Nephrol. 2007;18:2172-9.

26. Bakkaloglu SA, Warady BA. Difficult peritonitis cases in children undergoing chronic peritoneal dialysis: relapsing, repeat, recurrent and zoonotic episodes. Pediatr Nephrol. 2015;30:1397-406.

27. Dahlinghaus EK, Neu AM, Atkinson MA, Fadrowski JJ. Hemoglobin level and risk of hospitalization and mortality in children on peritoneal dialysis. Pediatr Nephrol. 2014;29:2387-94.

28. Borzych-Duzalka D, Bilginer Y, Ha IS, Bak M, Rees L, Cano F, et al Management of anemia in children receiving chronic peritoneal dialysis. J Am Soc Nephrol. 2013;24:665-76.

29. Civilibal M, Caliskan S, Oflaz H, Sever L, Candan C, Canpolat N, et al. Traditional and "new" cardiovascular risk markers and factors in pediatric dialysis patients. Pediatr Nephrol. 2007;22:1021-9.

30. Bakkaloglu SA, Saygili A, Sever L, Noyan A, Akman S, Ekim M, et al. Assessment of cardiovascular risk in paediatric peritoneal dialysis patients: a Turkish Pediatric Peritoneal Dialysis Study Group (TUPEPD) report. Nephrol Dial Transplant. 2009;24:3525-32.

31. Bakkaloglu SA, Buyan N, Funahashi $T$, Pasaoglu $H$, Elhan $A H$, Hasanoglu E, et al. Adiponectin levels and atherosclerotic risk factors in pediatric chronic peritoneal dialysis patients. Perit Dial Int. 2005;25:35761.

32. Borzych D, Rees L, Ha IS, Chua A, Valles PG, Lipka M, et al. The bone and mineral disorder of children undergoing chronic peritoneal dialysis. Kidney Int. 2010;78:1295-304

33. Gonçalves C, Sandes AR, Azevedo S, Stone R, Almeida M. Complicações da transplantação renal em idade pediátrica. Acta Med Port. 2013;26:517-22

34. Rees L. Long-term outcome after renal transplantation in childhood Pediatr Nephrol. 2009;24:475-84.

35. Mencarelli F, Kiepe D, Leozappa G, Stringini G, Cappa M, Emma F. Growth hormone treatment started in the first year of life in infants with chronic renal failure. Pediatr Nephrol. 2009;24:1039-46.

36. Rees L, Azocar M, Borzych D, Watson AR, Büscher A, Edefonti A, et al Growth in very young children undergoing chronic peritoneal dialysis. J Am Soc Nephrol. 2011;22:2303-12.

37. Santos F, Moreno ML, Neto A, Ariceta G, Vara J, Alonso A, et al. Improvement in growth after 1 year of growth hormone therapy in wellnourished infants with growth retardation secondary to chronic renal failure: results of a multicenter, controlled, randomized, open clinical trial. Clin J Am Soc Nephrol. 2010;5:1190-7.

38. Warady BA, Belden B, Kohaut E. Neurodevelopmental outcome of children initiating peritoneal dialysis in early infancy. Pediatr Nephrol. 1999;13:759-65.

39. Laakkonen $\mathrm{H}$, Lönnqvist $\mathrm{T}$, Valanne L, Karikoski J, Holmberg C, Rönnholm K. Neurological development in 21 children on peritoneal dialysis in infancy. Pediatr Nephrol. 2011;26:1863-71. 